\title{
Highlights of recent clinically relevant papers
}

\section{Oesophageal incompetence following prosthetic laryngoplasty}

In this study Safia Barakzai and colleagues from the UK and Ireland describe a complication observed endoscopically in horses following prosthetic laryngoplasty (LP).

This case series reported on 5 horses that had previous LP. Four horses which were part of a larger prospective study were observed to have saliva emanating from their upper oesophageal opening during endoscopic examination 21-58 months after LP. One other horse was referred with clinical signs of severe upper oesophageal obstruction 2 months after LP. Oesophageal reflux was noted endoscopically at rest in one horse and during exercise in 4 horses. Post operative coughing was reported in all 5 horses and 2 horses coughed severely when eating. Necropsy findings on the horse referred with signs of oesophageal obstruction were dilation of the proximal oesophagus associated with food impaction and fibrosis and thickening of the left cricopharyngeus and thyropharyngeus muscles.

The authors concluded that iatrogenic damage to the caudal pharyngeal constrictor muscles, the intrinsic musculature of the upper oesophagus, or their innervation, or damage to the peri-oesophageal fascia or oesophageal adventitia may cause upper oesophageal incompetence in horses following LP.

\section{Equine coronavirus infection}

In this retrospective study $C$. Fielding and colleagues in the USA describe the epidemiological and clinical features of two outbreaks of equine coronavirus (ECOV) infection that were associated with a high case fatality rate.

The epidemiological and clinicopathological findings, and faecal viral load from 14 miniature horses and one miniature donkey that tested faecal positive for ECoV from two related disease outbreaks were reported in this study. Of these 15 animals, 4 died or were subjected to euthanasia. One animal that subsequently died had severe hyperammonaemia and signs of encephalopathic disease. Faecal viral load (ECoV genome equivalents per gram of faeces) were found to be significantly higher in the nonsurvivors compared with animals that survived.

Equine coronavirus had a higher case fatality rate in this group of miniature horses than previously reported in other outbreaks of varying breeds. The authors suggested that hyperammonaemia could contribute to signs of encephalopathic disease, and the faecal viral load might be of prognostic value in affected horses.

\section{Hospitalised horses with botulism}

A. Johnson and colleagues in the USA have recently published their study evaluating the outcome of adult horses with botulism treated at a veterinary hospital.

Reported survival rates in outbreak situations are low; however, many horses in outbreaks do not receive treatment. In this retrospective study, the authors hypothesised that adult horses treated at a veterinary hospital would have improved survival compared with outbreak situations. The authors also also aimed to identify predictors of nonsurvival.

Medical records of all horses $>6$ months of age with a final diagnosis of botulism admitted to a veterinary teaching hospital over a 25-year period were reviewed and case details including admission and hospitalisation were recorded. Associations between variables and nonsurvival were identified using logistic regression. Two multivariable models were developed pertaining to the information available at admission and the clinical findings during hospitalisation. Ninety-two records met inclusion criteria.

Higher rectal temperature and dysphagia observed at admission were found to increase the odds of survival, as did treatment with antitoxin. Horses with abnormal respiratory effort or inability to stand had decreased odds of survival. Overall survival was $48 \%$ but was significantly higher (67\%) for horses that arrived standing, and even higher (95\%) for horses that remained able to stand throughout hospitalisation. Complications occurred in $62 \%$ of horses but were not associated with nonsurvival. The authors concluded that horses that lose the ability to stand have a poor chance of survival. Complications are common in treated horses but do not reduce survival.

\section{Short-term complications after colic surgery}

In this retrospective case-control study Deanna Gazzerro and colleagues in the USA compared the occurrence of short-term complications and short-term outcome associated with complications after colic surgery in geriatric and mature horses.

Medical records of horses that recovered from general anaesthesia after colic surgery were reviewed and these horses were divided into two groups. The geriatric group consisted of 78 horses aged $\geq 20$ years and the mature nongeriatric group consisted of 156 horses aged 4-15 years.

Post operative complications evaluated included post operative reflux (POR), diarrhoea, inappetence, fever, leukopenia, incisional infection, incisional dehiscence, thrombophlebitis, colic, repeat celiotomy, pneumonia and laminitis. Short-term outcome (alive vs. dead at hospital discharge) of geriatric and mature horses with these complications were compared and data was analysed.

Geriatric horses were found to be more likely to have a small intestinal strangulating lesion than mature horses and a higher proportion of geriatric horses had POR and inappetence. There was no difference in the proportion of geriatric and mature horses with small intestinal strangulating lesions having POR. The authors concluded that geriatric and mature horses have similar occurrences of short-term complications and short-term outcomes associated with complications after colic surgery.

\section{Back dimension changes in sports horses}

In some horses major back dimension changes over time have been observed. Work type, skeletal maturity, nutrition and saddle fit can all influence the speed of these changes. This study by Line Greve and Sue Dyson aimed to quantify these changes. 
This prospective, longitudinal study had a sample population of 104 sports horses in normal work. Thoracolumbar dimensions/symmetry were measured at predetermined sites every second month over one year and weight, work and saddle management changes were recorded. Descriptive statistics, and univariable and multiple mixed effects linear regression were performed to assess the association between management changes, horse-saddlerider factors and back dimension changes. Complete data was available for 63/104 horses, including an equal number of horses used for dressage, showjumping, eventing and general purpose, in four age groups: 3-5 years, 6-8 years, 912 years and $\geq 13$ years.

Considerable variations in back dimensions were recorded over one year. In the multivariable analysis, the presence of gait abnormalities at initial examination and back asymmetry were significant and had a negative effect on changes in back dimensions. Subsequent improved saddle fit, similar or increased work intensity, season (summer vs. winter) and increased bodyweight retained significance, having positive effects on changes in back dimensions. The authors concluded that quantifiable changes in back dimensions occur throughout the year. Saddle fit should be reassessed professionally several times a year, especially if there has been a change in work intensity.

\section{Paired measurement of ACTH concentration}

David Rendle and colleagues in the UK and USA performed this study to determine whether the mean of two measurements of adrenocorticotropic hormone (ACTH) concentration is more reliable in assessing pituitary pars intermedia dysfunction (PPID) than a single measurement.

Paired ACTH measurements were performed on 148 occasions from 124 horses being investigated for PPID, 90 occasions from 76 horses with PPID that were receiving treatment with pergolide, and 63 occasions from 50 horses in which there was no clinical suspicion of PPID. Histological examination of the pars intermedia was performed in 67 of the untreated horses. Outcome of testing using single and the mean of paired samples was compared directly and both methods were compared against histology, which was considered the gold standard.

Paired ACTH measurement differed from the single measurement in 6 of 211 cases, all of which had equivocal initial ACTH concentrations between 20 and $39 \mathrm{pg} / \mathrm{ml}$. Using histology as the gold standard, optimal sensitivity and specificity for diagnosing PPID were 69.4 and $80.9 \%$, respectively, for a single measurement and 72.2 and $76.2 \%$, respectively, for paired measurements. The area under the receiver operating characteristic curve was 0.72 and 0.73 for single and paired measurements compared with histopathologic diagnosis, respectively.
The authors concluded that paired measurement of $\mathrm{ACTH}$ concentration offers no advantage over a single measurement.

\section{Laser assisted modified Forssell's procedure}

In this retrospective case series, Petrisor Baia and colleagues at Lovisiana State University, USA, report on the long-term outcome after laser assisted modified Forssell's procedure in cribbing horses. The prevalence of post operative complications and the risk factors associated with outcome are also discussed.

Case details including preoperative duration of cribbing, post operative complications and outcome were obtained from the medical records of 119 horses that had laser assisted modified Forssell's procedure.

Follow-up was available for 90 horses; 76 had stopped cribbing for more than one year. The median relapse time in the 14 horses that resumed cribbing was 6 months. Preoperative cribbing duration was significantly associated with an increased probability of unsuccessful outcome when horses cribbed for $>3$ years before surgery. Post operative complications (haematoma, seroma, infection and prolonged drainage) occurred in $22.2 \%$ of horses.

The authors concluded that laser assisted modified Forssell's is a very effective (84.4\%) surgical treatment of cribbing behaviour in horses. Preoperative cribbing duration was identified as a significant risk factor associated with unsuccessful surgical outcome.

S. WRIGHT

EVE Editorial Office

\section{References}

Baia, P., Burba, D.J., Riggs, L.M. and Beaufrere, H. (2015) Long term outcome after laser assisted modified Forssell's in cribbing horses. vet. Surg. 44, 156-161.

Barakzai, S.Z., Dixon, P.M., Hawkes, C.S., Cox, A. and Barnett, T.P. (2015) Upper esophageal incompetence in five horses after prosthetic laryngoplasty. Vet. Surg. 44, 150-155.

Fielding, C.L., Higgins, J.K., Higgins, J.C., Mclntosh, S., Scott, E., Giannitti, F., Mete, A. and Pusterla, N. (2015) Disease associated with equine coronavirus infection and high case fatality rate. J. Vet. Intern. Med. 29, 307-310.

Gazzerro, D.M., Southwood, L.L. and Lindborg, S. (2015) Short-term complications after colic surgery in geriatric versus mature nongeriatric horses. Vet. Surg. 44, 256-264.

Greve, L. and Dyson, S. (2015) A longitudinal study of back dimension changes over 1 year in sports horses. Vet. J. 203, 65-73.

Johnson, A.L., McAdams-Gallagher, S.C. and Aceto, H. (2015) Outcome of adult horses with botulism treated at a veterinary hospital: 92 cases (1989-2013). J. Vet. Intern. Med. 29, 311-319.

Rendle, D.I., Duz, M., Beech, J., Parkin, T. and Durham, A.E. (2015) Investigation of single and paired measurements of adrenocorticotropic hormone for the diagnosis of pituitary pars intermedia dysfunction in horses. J. Vet. Intern. Med. 29, 355-361. 